\title{
XXVI.
}

Aus der psychiatrischen Klinik der Königlichen Charité (Prof. Jolly).

\section{Anatomische Untersuchung des unter dem Titel „Ueber einen diagnostisch unklaren Fall von Er- krankung des Nervensystems" von Professor Dr. Westphal in den Charité-Annalen (XIV. Jahrgang 1889) verüffentlichten Falles.}

Von

\section{Dr. A. Westphal,} Assistenzarzt.

(Hierzu Taf. XIII. und XIV.)

„Ueber einen diagnostisch unklaren Fall von Erkrankung des Nervensystems", unter diesem Titel hat mein Vater in den Charité-Annalen (XIV. Jahrgang, 1889) die Krankengeschichte eines Arbeiters Frommbolz veröffentlicht. Der Fall bot in seinen klinischen Erscheinungen und seinem Verlauf so viel Eigenthümliches dar, dass W. meinte, von der Stellung eiper bestimmten Diagnose Abstand nehmen zu müssen. Am 27. December 1889 starb Frommholz auf der chirurgischen Klinik an einer Phlegmone perinei unter den Erscheinungen von Herzsch wäche. Das Rückenmark, die peripherischen Nerven und Muskeln, welche damals von Herrn Prof. Siemerling der Leiche entnommen und in Müll e:'scher Flüssigkeit konservirt wurden, sind jetzt von mir mikroskopisch untersucht worden.

Das Resultat dieser Untersuchung möchte ich, da es viel Interessantes darbietet und geeignet ist, einiges Licht auf den bisher dunklen Fall zu werfen, in Folgendem mittheilen. Ein kurzer Ueberblick über die wichtigsten klinischen Erscheinungen dürfte wohl vorher zur 
leichteren Orientirung hier am Platze sein; in Betreff der speeiellen Verhältnisse verweise ich auf die von meinem Vater veröffentlichte Krankheitsgeschichte (l. c.).

Es handelt sich um einen 28jährigen, dem Alcoholgenuss in hohem Grade ergebenen Arbeiter, Frommholz. Dieser erkrankte im December 1885 unter allmälig zunehmender Schwäche der Beine, der sich bald Schwellungen der unteren Extremitäten und des Gesichts zugesellten. Bei der Aufnahme (1. Mai 1886) fiel zunächst das enorme Volumen der unteren Extremitäten auf, welches hauptsächlich durch die sehr starke Entwickelung des Fettpolsters und nur zum Theil durch ein Hautödem bedingt war. Es bestand hochgradige allgemeine Adipositas. Die active Beweglichkeit der unteren Extremitäten war fast vollkommen aufgehoben, auch die passiven Bewegungen erschienen erheblich beschränkt.

Die sehr eigenthümliche Configuration der oberen Extremitäten war bedingt durch Verdickungen der Ober- und Unterarme und vornehmlich der Hände, in Verbindung mit Oedemen und partiellen Atrophien. Active und passive Beweglichkeit war auch in den oberen Extremitäten deutlich vermindert.

Die electrische Untersuchung ergab stellenweis einfache quantitative Herabsetzung der Erregbarkeit, zum Theil bis zum Erlöschen derselben, an einzelnen Stellen Entartungsreaction. (Muskeln des Daumenballens, Opponens pollicis.) Sowohl obere wie untere Extremitäten zeigten ausgesprochene Sensıbilitätstörungen. Die Sehnenphänomene waren aus mechanischen Gründen nicht zu erzielen.

An den Gebirnnerven konnte keine Abnormität constatirt werden.

Das Gesicht sah eigenthümlich gedunsen aus, batte einen stupiden Ausdruck.

Psychisch war deutliche Demenz vorhanden.

In den ersten 4 Wochen des Krankenhausaufenthalts bestand ein unregelmässiges, intermittirendes Fieber, welches mit hoher Pulsfrequenz verlief, ohne dass eine Ursache für dasselbe nachweisbar gewesen wäre.

Im ferneren Krankheitsverlauf war eine sehr beträchtliche Besserung verschiedener Krankheitssymptome bemerkenswerth.

Vor Allem waren die Lähmungserscheinungen beträchtlich zuräckgegangen, sowohl an den oberen, wie an den zuerst noch stärker betroffenen Unterextremitäten.

Vom 20. December 1887 steht in der Krankengeschichte notirt: Patient ist jetzt im Stande, mit Unterstützung oder mittelst eines Stockes, 
den er in die rechte Hand nehmen kann, durch's Zimmer zu gehen. Das Gesammtresultat der electrischen Untersuchung war jetzt eine beträchtliche Herabsetzung der Erregbarkeit, besonders in den Streckern der Hand und in den kleinen Handmuskeln.

Die Störungen der Sensibilität waren vollkommen gesehwunden; die Oedeme bis auf geringe Reste zurückgegangen. Die von Anfang an bestehende Adipositas hatte hingegen im Verlauf des Krankenhausaufenthaltes noch beträchtlich zugenommen. Das am 18. Februar 1887, 255 Pfd. betragende Gewicht des Kranken war im September 1887 bis auf 332 ! Pfd. angestiegen - eine wahre Polysarcie.

Die Demenz des Patienten hatte ebenfalls einem etwas einsichtsvolleren Wesen Platz gemacht.

Unverändert war die eigenthümliche Deformität der Hände geblieben.

In diesem Zustande wurde F. am 21. Februảr 1889 aus der Charité entlassen.

Am 7. December 1889 wurde er wieder wegen einer Phlegmone des Perineum auf der chirurgischen Station aufgenommen, woselbst er am 27. December 1889 unter den Erscheinungen von Herzschwäche starb.

Wir haben also Alles in Allem eine chronisch verlaufende Krankheit vor uns, die charakterisirt war durch atrophische Lähmung der Extremitäten, Sensibilitätsstörung, Oedembildung, hochgradige Adipositas, zeitweilig auftretendes Fieber und Demenz, Symptome, die sich sämmtlich allmälig mebr oder weniger zurückbildeten - so dass man in Berücksichtigung des ätiologischen Moments wohl an eine Alkoholneuritis mit Ausgang in unvollkommene Genesung denken konnte. Auffällig blieben bei dieser Annahme immer die Deformität der Hände und die enorme Fettsucht.

Die anatomische Diagnose lantet: Erysipelas, Phlegmone perinei, Oedema pulm., Hyperplasia pulpae lienis, Nephritis parench. recens., Gastritis parench. et catarrhalis., Hyperplasia tela adiposae permagna, Oedema Arachnoidis, Dilatatio et hypertrophia cordis, Induratio rubra pulm.

Aus dem Sectionsprotocoll hebe ich Folgendes hervor: Das Fettpolster ist sehr beträchtlich vermehrt. Die Stärke schwankt von 4-9 Ctm. an den verschiedenen Körperstellen. Die Mnskulatur zeigt überall Entwickelung von Fettgewebe zwischen den Muskelbündeln, aber in mässiger Menge.

Das Herz ist grösser als die Faust, der rechte Vorhof stark ausgedehnt. Pericardiam ist fottreich, Klappen intact.

Die Lungen zeigen alto Verwachsungen, sind ödematös.

Mesenterium und 0 mentum sebr fettreich. 
Milz 16, 11, $5 \mathrm{Ctm}$. gross, brüchig, Follikel deatlich trübe: Pulpa vermehrt.

Nierenkapsel enorm fettreich, die Rinde der Nieren ist sebr breit, getrübt, rechts mehr wie links.

Magenscbleimhaut ist mit Schleim bedeckt, im Fundus lebhaft geröthet, zum Theil hämorrhagisch, gefaltet, nach dem Duodenum zu getrübt.

Leber enorm gross und schwer, Acini sehr gross, in den peripherischen sebr deutliche Fetinfiltration.

Pancreas sebr derb, fest, bart, gelappt, $z$ wischen den Lappen schieben sich von aussen her Fettläppchen hinein.

An der linken Seite des Dammes eine Wunde, welche in eine Abscesshöhle bineinführt.

Schädeldach sehr dick, gestreckt gebant, Diploe sehr breit und blutrejch. In den Sin. long. viel flässiges Blut und Speckgerinnsel.

Dura auf der Innenfläche unverändert. Die Arachnoides weisslich, verdickt, etwas ödomatös. Gyri am Stirnlappen schmal, breit am Scheitellappen. Die Sulci zeigen blasiges Dedem.

Gefässwandungen an der Basis elwas, aber gleichmässig verdickt, Gefässe mit flüssigem Blut gefüllt. Gew. $1280 \mathrm{Grm}$. Ventrikel, Tela, Markmass $\theta$ zeigen nichts Auffallendes. folgen:

Die Ergebnisse der mikroskopischen Untersuchung lasse ich nunmehr

Rückenmark (Färbung mit Carmin, Nigrosin und nach Weigert). Halsanschwellung: Die Untersuchung der grauen Substanz ergiebt, dass die Zahl der Ganglienzellen in beiden Vorderbörnern eine verschieden grosse ist. Die vergleichende Zählung mit normalen Präparaten ergiebt im Ganzen eine entschiedene Verminderung der Zahl der Ganglienzellen. Diese Verminderung ist anf verschiedenen Schnitten eine verschieden grosse; die Zahl beträgt etwa $1 / 2$ bis $1 / 4$ der Anzahl der Ganglienzellen in normalen Vorderbörnern. (Fig. 7 u. 8, Taf. XIV.) geben ron diesem Verbältniss eine schematische Uebersicht.) In der äusseren Gruppe sind die Ganglienzellen durchweg gut entwickelt, zeigen namentlich weit zu verfolgende Fortsätze, Kerne und Kernkörperoben. Ebenso die innere Gruppe; in der mittleren Gruppe finden sich an einzelnen Präparaten einige Zellen, welche ein kugliges Aussehen haben und deren Fortsätze sich nur eine ganz kurze Strecke weit verfolgen lassen (Fig. 6, Taf. XIII.). Die Grundsubstanz weist keine abnorme Gefässentwickelung oder Spinnenzellen auf.

Die vorderen Wurzeln sowohl in ihrem intra- wie extramedullären Verlauf sind intact.

Sonst keine Veränderungen nachweisbar.

Unterster Halstheil: In dem vordersten Ende des eigentlichen Vorderhorns sieht man gut entwickelte Ganglienzellen, das Seitenhorn weist hingegen eine Verminderung und Atrophie derselben auf. Es finden sich hier bei schwacher Vergrösserung (Hartnack S. 20.3 ), nur in wenigen Schnitten, noch ganz kleine knopfförmige Gebilde. Bei stärkerer Vergrösserung erweisen 
sich diese als Reste von Ganglienzellen; in manchen sind die Fortsätze noch eine Strecke weit, zuweilen korkenzieherförmig gestaltet, zu verfolgen. Kerne und Kernkörperchen sind in den atrophischen Zellen nicht mehr zu erkennen.

Die Clarke'schen Säulen zeigen in ihrem proximalen Ende ausserordentlich kleine Ganglienzellen, an manchen Präparaten vermisst man sie auf einer Seite ganz. In den Vorderhörnern sind die Gefässe stark mit Blut gefüllt, und man sieht in der Umgebung der Wandung zuweilen auf einer. kurzen Strecke frische Blutaustritte in das Gewebe. Die vorderen und hinteren Wnrzeln lassen keine Veränderangen erkennen, ebensowenig dio Faserung der grauen Substanz.

Oberer Dorsaltheil: Die Zahl der Zellen in den Vorderhörnern ist entschieden vermindert, es finden sich die bereits besohriebenen veränderten Zellen. Die Zellen in den Clarke' schen Säulen sind gut entwickelt, nur selten sind einige von sehr geringem Umfang, doch sieht man bei Vergleich mit normalen Präparaten auch in diesen derartige kleine Zellen in den Clarke'schen Säulen. Vordere und hintero Wurzeln, sowie Faserung der grauen Substanz sind gut. Dieselben Verhältnisse finden sich im mittleren Dorsaltheil.

Unterer Dorsaltheil (Uebergang in den Lendentheil). Die Vordersäulen sind insofern verschieden, als die Ganglienzellen der einen Seite gewöhnlich in grossen periceliulären Lymphräumen liegen, während diese Lymphräume auf der anderen Seite nicht vorhanden sind. Die Zahl der Ganglienzellen ist vermindert, ein grosser Theil auch wieder atrophisch. Derselbe Untersohied lässt sich bei den Clarke'schen Säulen constatiren, doch kann man hier von einer Verminderung der Zellen nicht sprechen, wohl aber finden sich auch hier eine Anzahl atrophischer Zellen.

Oberster Lendentheil: Die Zellen liegen beiderseits in grossen pericellulären Lymphräumen und wenn anch einzelne derselben gut erhalten sind, so zeigt doch eine nicht geringe $\mathrm{Zahl}$ die bereits beschriebenen Veränderungen. Bei manchen Ganglienzellen erscheint der Zellleib von netzartiger Structur, derselbe ist von Hohlräumen (Vacuolen) durehsetzt. Die Zahl der Zellen ist kaum vermindert.

Oberste Lendenanschwellung: Auch hier ist die Zahl der Zellen nicht vermindert, einzelne Schnitte allerdings zeigen eine geringe Anzahl, während andere verglichen mit normalen keine Abweichung erkennen lassen. Bei einer grossen Anzahl von Zellen jedoch lassen sich Veränderungen nachweisen, die sich beziehen auf Verlust der Fortsätze, Zerfall des Zellleibs und Vacuolenbildung. Bei manchen Zellen ist der Zellleib blasig aufgetrieben; besonders anffällig ist dies an einzelnen Stellen, wo die eine Hälfte der Zelle in eine ganz homogene Masse umgewandelt ist, dieser Theil setzt sich scharf wie dnrch einen Spalt von der anderen Hälfte ab. In der mehr lateralwärts gelegenen Gruppe haben die Kernkörperchen in den Weigert-Prüparaten eine intensiv schwarze Färbung angenonmen. Neben diesen veränderten Zellen findet sich aber eine Anzahl, an denen man keine Veränderung naohzuweisen im Stande ist. 
Die untere Lendenanschwellung bietet dasselbe Bild; vordere und hintere Wurzeln sind auch hier durchaus intact.

Resümiren wir kurz das Resultat der Untersuchung des Rückenmarks: Die weisse Substanz ist intact, die Ganglienzellen in den Vorderhörnern der grauen Substanz zeigen theilweise Degeneration - am meisten ausgesprochen ist dieselbe in der Halsanschwellung, weniger in der Lendenanschwellung. In ersterer ist es auch zu einer Abnahme der Zahl der Zellen gekommen.

Vordere und hintere Wurzeln sind intact.

Die Untersuchung der in Müller'scher Flüssigkeit gebärteten Muskelstückchen (Färbung mit Carmin und Hämatoxylin) ergab im Einzelnen Folgendes:

M. biceps femoris. Der Muskel zeigt normales Aussehen.

Die Fasern nicht rundlich, sondern polygonal, nicht die geringste Vermebrung des interstitiellen Gewebes. Die Gefässwandungen sind nicht verdickt. -

Ebenso bietet M. semitendinosus durchaus normales Verhalten.

M. peroneus brevis. Die Fasern haben ein sebr wechselndes Kaliber, mehr rundliche Form. Ein grosser Theil des Muskels ist umgewandelt in Fett. Die grossen Fasern, mit einem Durchschnitt von $93 \mu$, zeigen selten Vacuolen und Spaltbildung. Daneben ganz kleine atrophische Fasern mit einem Durchschnitt von ca. $13 \mu$. Die Gefässwandungen sind etwas verdickt. Neben den stark verfetteten Partien finden sich kleine Stellen im Muskel, die noch gut erbaltene polygonale Fasern aufweisen. Noch andere Partien enthalten hochgradig atrophische Fasern, Reste, die kaum noch als Fasern zu erkennen sind. Dieselben liegen in einem stark verbreiterten, derben Bindegewebe. An einzelnen Stellen liegen haufenweis Kerne, hier hat auch eine reiche Gefässentwicklung Platz gegriffen. Einzelne im Muskel verlanfende Nervenstämmohen zeigen hochgradige Atrophie. Der Querschnitt besteht fast nur aus kleinen Ringen. In einzelnen ist noch ein Axencylinder und eine kleine Markumbüllung sichtbar.

M. peroneus longus. Die Verfettung ist nicht so bochggradig, wie im Peroneus brevis. Es finden sich aber auch hier Bezirke, welche weiter nichts aufweisen, als Fettzellen. Die Muskelfasern sind hypervoluminös, ca. $116 \mu$. Auf dem Längsschnitt lassen die meisten Fasorn noch elne ausgesprochene Querstreifung erkénnen, manche verlaufen ganz wellig, und der Inhalt der Muskelfaser erseheint dann wie zusammen geschoben, in Ringen angeordnet. Spaltbildung tritt auch auf dem Längsschnitt dentlich hervor, an anderen Stellen auch dichotomische Theilang. Vacuolenbildung deutlich rorhanden. An manchen Fasern sind die Kerne in Reihen angeordnet. Das interstitielle Gewebe ist leicht vermehrt, manchmal mit Kernwucherung.

M. rectus femoris. Setzt sich aus grossen, fast durchweg runden, ca. $116 \mu$ betragenden Fasern zusammen. Die kleinsten Fasern betragen ca. 
$27 \mu$, eigentlich atrophisehe Fasern sind nicht vorhanden. Vacuolenbildung ist hänfig, Spaltbildungen vereinzelt and rur angedentet. Die Gefässwan= dungen verbreitert, in der unmittelbaren Nähe eines grösseren Gefässes, eine frische Blatung, die sich ziemlich weit in's Gewebe verfolgen lässt. Das interstitielle Gewobe ist etwas vermehrt und kernreich. Die Zahl der Muskelkerre beträgt $10-15$.

M. vastus femoris (Fig. 1, 2, 3, Taf. XIII.). Während ein grosser Theil des Muskels ein normales Verhalten aufweist (Fig. 1) sind andere Stellen verändert und man kann den Uebergang von gesunden zu kranken Partien an ein und demselben Präparat deutlich erkennen. Figuren 1, 2 und 3 sind nach dicht bei einander liegenden Stellen dieses Muskels gezeichnot. Es finden sich neben den normalen Partien, (Fig. 1) Stellen, wo die Muskelfasern hypervoluminös, rundlich sind (Fig. 2), Spaltbildnngen zeigen und nicht mehr die scharfe Zeichnung der Cohnheim ${ }^{2}$ schen Felder erkennen lassen. Der Durchschnitt der Fasern beläuft sich hier auf $138-158 \mu$. Unmittelbar hieran liegen vollkommen atrophische Stellen (Fig. 3), in denen die Fasern ausserordentlich geschrnmpt, das Bindegewebe sehr vermehrt und kernreich ist, und sich intexstitielle Einlagerung von Fettzellen findet. Die Nervenstämmchen sind stark degenerirt, die Gefässwandungen verdickt, auch hier findet sich in der Nähe eines grösseren Gefässes Blutaustritt in's Gewebe.

M. solens. Es finden sich durch den ganzen Muskel verbreitet, der sich zum grossen Theil aus polygonalen Muskelfasern zusammensetzt, deren Durobmesser $z$ wischen 69 und $93 \mu$ schwankt, einzelne rundliche entschieden hypervoluminöse Fasern. Dieselben heben sich bei der Pikrocarminfärbung dentlich dadurch $a b$, dass sie die Farbe nicht so gut angenommen haben und mehr gelbglänzend hervortreten. Der Durchmesser einzelner Fasern heträgt 117-138 $\mu$. In manchen dieser grossen Fasern ist das Protoplasma in der Mitte zerfallen, hat dann eine Rosafärbung angenommen, in einzelnen ist eine Vacuolenbildung siohtbar.

Spaltbildungen sind bier nicht vorhanden. Die grossen Fasern sind rosenkranzartig mit Kernen besetzt, ca. 20 an einer Faser.

M. gastrocnemius. Der grösste Theil des Muskels ist in Fett umgewandelt; dazwischen sieht man noch einzelne Inseln, wo die Muskelfasern verhältnissmässig gut sind und andere Stellen, wo neben den bypervoluminösen sich stark atrophische Stellen finden, das interstitielle Gewebe stark vermehrt ist und Kernwucherung anfwoist. Hier findet man anoh Sarcolemm. Schläuche angefüllt mit Kernen, vereinzelt auch gelbliches Pigment. Vacuolenbildung ist häufig auch in nioht hypervoluminösen Fasern. Die Gefüsswandungen sind stark verdickt.

M. interosseus (Hand).

Die Fasern sind fast durchwog rundlich, das Caliber sohr versehieden, ïberwiegend bypertrophische Fasern mit einem Volumen von 132-158 die kieinsten Fasern haben einen Durchmesser von ca. $18 \mu$. In vielen Fasern beginnende oder vollendete Spaltbildung; die Spaltbildung ist an manchen Fasern sehr zahlreich, so dass die Faser in Streifen zerklüftet erscheint. In 
der Mitte, von der ans die Spaltung erfolgt, liegen gewöbnlicb einige Kerne. Vacuolenbildung ist sehr vereinzelt; das interstitielle Gewebe ziemlich beträchtlich vermehrt, sehr kernreicb. Die Gefässwandungen sind nicht verdickt. An manchen Stellen ist es bereits zur Bildung ron Fettzellen gekommen. Die Kerne des Sarcolemms sind vermehrt, in einer Muskelfaser 7 - 10 .

Thenar. Im Grossen und Ganzen bietet der Muskel dasselbe Bild wie der Peroneus longus. Die Gefässe sind ansserordentlich verdickt in ihren Wandungen, einige lassen gar kein Lumen mehr erkennen.

Hypothenar. Wäbrend ein Theil des Muskelballens ein normales Aussehen zeigt, haben in einem anderen die Fasern fast durchweg eine rnndliche Form angenommen, sind hypertrophisch, es finden sich Fasern von $117 \mu$. Eigentlich atrophische. Fasern sind nicht vorhanden. Das interstitielle Gewөbe sehr gering vermebrt. Die Zahl der Muskelkerne 5-8. Auf dem Längsschnitt sieht man einzelne grosse Fasern sich in zwei theilen.

M. opponens podlicis. Fasern darchweg hypervoluminös, ca. $116 \mu$. Spaltbildung und Vacuolen. Interstitielles Gowebe leicht vermehrt. Getässe in den Wandungen verdickt.

M, extensor dig.commun. (Hand) zeigt dasselbe Verbalten wie der Rectus femoris.

M. extensor dig. brevis (Hand). Hypervolnminöse Fasern, Vermehrung des interstitiellen Gewebes. Bild ähnlich wie beim Peroneus long.

M. flexor dig. sublimis. Während ein Theil des Muskels im Wesentlichen normale Structur zeigt und sich nor vereinzelte rundliche bypervoluminöse Fasern finden und das interstielle Gewebe nicht vermehrt ist; setzt sich ein anderer Theil aus deutlich hypervoluminösen rundlichen Fasern zusammen, mit leichter Vermehrung des interstitiellen Gewebes.

M. flexor dig. prof. ein Theil des Muskels normal; ein anderer weist deutlich hypervoluminöse, rundliche Fasern auf, mit leichter Vermebrung des interstitiellen Gewebes.

M. extensor carpi radialis zeigt ein gleiches Verbalten wio der Soleus. Es finden sich mehrere neuromusculäre Stämmchen.

M. biceps brachii gesund wie der Biceps fem., nur ganz vereinzelc (in einem Schnitt, eine Faser), stösst man auf eine Faser, welche hypervoluminös, die Färbang nicht angenommen hat.

Von den Nerven wurden mikroskopisch untersucht (Färbung nach Weigert, Carmin-und Haematoxylin-Färbung): N. cruratis (Muskelast), N. ischiadicus, N. tibialis post., N. Peroneus prof., $\mathrm{N}$. musoulo-cutanens, N. medianus, $\mathrm{N}$. ulnaris, $\mathrm{N}$. radialis.

N. oruralis (Muskelast). Im Ganzen normales Ausseben; es sind viele kleine Fasern vorhanden; in den aller meisten erkenni man noch deutlioh den kleinen Axencylinder, nur in einzelnen Bündeln finden sich Stellen, in denen die Structor der kleinen Fasern niobt mehr deutlicb za erkennen ist.

Keine Vermebrung des interstitiellen Gewebes, zeine Kernvermebrung. 
N. ischiadicus. Zwischen gut aussehenden grossen Fasern fanden sich Haufen, wo theilweise ganz kleine atrophische Fasern liegen, oder blosse Ringe. Die Kerne sind an diesen Stellen vermehrt. Die Gefässe zeigen deutliche Wandverdickung.

N. tibialis posticus (Fig. 5, Taf. XIII.) bietet das Bild höchster Degeneration dar, es findetsichim Gesichtsfeld kaum eine normale Faser - nur wenige, welche noch eine Markumhüllung zeigen. Das Mark ist aber zerfallen, zeigt keine concentrische Schichtang und meist fehlt der Axencylinder. Der grösste Theil des Nerven besteht aus sehr feinen Ringen, in denen sehr selten ein Pünktehen (atrophischor Axencylinder) sichtbar ist. Das interstitielle Gewebe ist reichlich vermehrt. Es besteht Kernwucherung. Die Wandungen der Gefässe, sowohl der des Epineuriums, als auch der im Endoneurium liegenden, sind verdickt.

N. peroneus prof. zeigt fast ebenso starke Atrophie wie der Tibialis posticus. Es finden sich hier in jedem Nervenbündel einige gut erbaltene Fasern.

N. museulo cutaneus mit durchweg grossen Fasern, wohl ausgebildeten Markscheiden und Axencylindern, ist völlig normal.

N. median us bietet ein ähnliches Bild wie der Ischiadicus, nur sind die Haufen der atrophischen Fasern hier bei Weitem nicht so gross. - Die Gefässwandungen sind deutlich verdickt.

N. ulnaris ist deutlich atrophisch. Die Mehrzahl der grossen breiten Fasern zeigt einen Zerfall des Marks, Schwund des Axencylinders, daneben finden sich viele kleine Ringe, in denen die Structur von Nervenfasern nicht mehr sichtbar ist. Leichte Vermehrung des interstitiellen Gewebes, Kernwucherung, Gefässverdickung. Die Veränderungen sind in einzelnen Bündeln stärker ausgesprochen, als in anderen.

N. radial is bietet das Aussehen eines normalen Nerven.

Wir sehen also, dass die mikroskopische Untersuchung des Rückenmarks, der Muskeln und der peripherischen Nerven deutliche Veränderungen ergeben hat. Am wenigsten ausgedehnt sind diese Veränderungen am Rückenmark. Sie beschränken sich hier auf die Ganglienzellen der Vorderhörner und der Clarke'schen Säulen. Diese Zellen sind in qualitativer und quantitativer Weise veränderts Die qualitativen Veränderungen bezieben sich auf kugliges oder knopfförmiges Aussehen der Zellen, Verlust oder schlechte Ausbildung der Fortsätze; in den am stärksten afficirten Zellen vermissen wir Kerne und Kernkörperchen. Der Zellleib mancher Zellen zeigt eigenthümliche netzartige Structur mit Vacuolenbildung, einige Zellen sind blasig aufgetrieben. Die quantitativen Veränderungen der Zellen sind besonders prägnant im Halstheil des Rückenmarks hervortretend. Auf den ersten Blick sieht man bier eine auffallende Verminderung der Zabl der Ganglienzellen. 
Bei vergleichender Zählung mit normalen Präparaten findet man die Zahl der Zellen in verschiedenen Schnitten bjs auf den vierten Theil der normalen Anzahl reducirt (vergl. Fig. 7 und 8, Taf. XIV).

Im untersten Theil der Halsanschwellung fanden wir die Gefässe stark mit Blat gefüllt, in der Ungebung der Gefässe vereinzeite kleine Blutungen in das Gewebe.

Alles in Allem bot demnach das Rückenmark ein Bild dar, wie wir es bei leichten Graden von Poliomyelitis anterior chronica zu finden gewohnt sind. Ausserdem trat uns ein gewisser Grad von Zellenverarmung und Degeneration in dem proximalen Ende der Clarke'schen Säulen entgegen.

Gehen wir nun von dem nervösen Centralorgan des Rückenmarks weiter nach der Peripherie za, so können wir zunächst constatiren, dass die vorderen und hinteren Wurzeln sowohl in ihrem intra- wie extramedullären Verlauf durchaus normal sind.

Ganz andere Verbältnisse boten die zur Untersuchung gelangten peripherischen Nerven dar, wir finden hier alle Jebergänge von den Bildern schwerster Degeneration (Fig. 5, Taf. XIII.) bis za denen völlig normaler Nerven.

Die degenerativen Verändernngen betreffen sowohl das nervöse, als auch das interstitielle Gewebe.

Vorwiegend sind die parenchymatösen Veränderungen, sie bieten die bekannten Erscheinungen der degenerativen Neuritis: Fehlen des Axencylinders, Schwund oder Zerfall des Marks, welches seine concentrische Schichtung verloren hat.

Die interstitiellen Vorgänge bestehen in leichterer oder stärkerer Vermehrung des interstitiellen Gewebes mit Kernwucherung. Auch die Gefässe, sowohl die des Epi- wie des Endoneuriums sind verändert und in ihren Wandungen verdickt.

Nicht weniger erbebliche und ausgedehnte Veränderungen wie die Nerven, bot die Muskulatur dar. Auch die Muskeln zeigen alle Grade der Degeneration von den leichtesten bis zu den schwersten Formen, neben einzelnen Muskeln (M. biceps fem., semitendinosus), die völlig normal erscheinen.

Wohl die augenfälligste Veränderung ist die Volumsverschieden. heit der einzelnen Muskelfasern; wir finden neben grossen hypervoluminösen (Erb) Fasern, ganz kleine atrophische Fasern vor. Diese Unterschiede treten uns einerseits bei der Vergleichung der verschiedenen Muskeln sehr deutlich entgegen, andererseits finden wir in ein und demselben Muskel neben stark hypertrophischen Stellen solche 
mit ganz atrophischen Fasern vor, und neben diesen veränderten Partien wiederum Bezirke mit normalen Fasern (M. vastus femoris, Taf. XIII., Fig. 1, 2 und 3). Die grössten gemessenen Fasern betrugen $158 \mu$, die kleinsten 13-18 $\mu$, diese sehr erhebliche Differenz fand sich $u$. A. auch in ein und demselben Muskel (M. interosseus manus, Fig 4, Taf. XIII.).

Ebenso prägnant wie die Veränderungen in der Grösse sind die Formveränderungen der einzelnen Muskelfasern. Dieselben zeigen nicht mehr die polygonale Form normaler Fasern, sondern eine mehr oder weniger starke Abrundung bis zu völliger Kreisform. Diese Abrundung betrifft sowohl die grossen wie die kleinen Fasern; auch hier finden sich wieder mitunter in einem Muskel neben diesen veränderten abgerundeten normale polygonale Fasern vor (Fig. 1, Taf. XIII.).

Die Muskelkerne zeigen an einzelnen Stellen eine einfache Vermehrung, an anderen Stellen eine bemerkenswerthe Anordnung in Reihen, wieder an auderen Fasern ist eine rosenkranzartige Anord. nung zu constatiren. In einzelnen Fasern, besonders in denen, in welchen die gleich zu erwäbnenden Spaltbildungen vorbanden waren, fanden sich auch central gelegene Kerne. Schliesslich wurden vereinzelt, vom Muskelinhalt entleerte Sarcolemmschläuche, angefüllt mit Kernen, beobachtet. Von den Veränderungen des Inhalts der Muskelfasern müssen wir als sehr häufige die Spaltbildungen hervorheben. Dieselben fanden sich, allerdings in verschiedener Zahl and Deutlichkeit, in der grössten Zahl der untersuchten Muskeln vor, besonders schön und deutlich in den grossen hypervoluminösen Fasern (Fig. 4, Taf.XIII.). An Längsschnitten beobachten wir an einzelnen Stellen dichotomische Theilung der Fasern.

Schliesslich zeigte das Protoplasma der Muskelfasern, ebenfalls in recht verschiedener Zahl und Ausbildung, theils Vacuolenbildung, theils eigenthümliche Zerfallserscheinungen, die sich besonders durch veränderte Empfänglichkeit der betreffenden Stellen gegen die angewandten Färbeflüssigkeiten documentirten.

Diese Veränderungen der Grösse und Gestalt der Muskelfasern, in Verbindung mit Kernvermehrung, Spalt- und Vacuolenbildungen stellten die wichtigsten parenchymatösen Veränderungen der erkrankten Muskulatur dar.

Wenden wir uns jetzt den interstitiellen Veränderungen zu. Dieselben bestehen vorwiegend in einer Vermehrung des interstitiellen Gewebes, mit bald stärkerer, bald schwächerer Ablagerung von Fettzellen. Diese Vermehrung des interstitiellen Gewebes lässt alle Grade von ganz leichter Wucherung bis zu Veränderungen erkennen, 
wo derbe, stark verbreiterte Bindegewebszüge, hochgradig atrophische - kaum noch als Fasern zu erkennende - Reste umgeben (Fig. 3). Hier findet sich denn auch starke Kernvermehrung, die mitunter zu Bildung grösserer Kernhaufen führt. Ebenso ist auch die Vermehrung des Fettgewebes stellenweis eine geringe, während an anderen Stellen die Fettwucherung eine sehr bedeutende ist, so dass ganze Partien des Muskels in Fett umgewandelt erscheinen. In diesen stärker degenerirten Theilen finden sich an den Gefässen deutliche Veränderungen, Verdickung der Wandungen und Blutaustritt in die Umgebung derselben.

Auch die intramusculär verlaufenden kleinen Nervenstämmchen bieten hier das Bild hochgradiger Atrophie dar. An einzelnen Stellen schliesslich finden sich die von Roth*) beschriebenen neuromuscnlären Stämmchen, welchen nach Siemerling**) eine pathologische Bedeutung nicht zukommt.

Ich habe im Vorhergehenden versucht, einen kurzen Ueberblick über die ausgedehnten pathologisch-anatomischen Veränderungen zu geben, welche uns entgegengetreten sind. Wir fanden im Rückenmark das Bild einer Poliomyelitis anterior chronica (mit Betheiligung der Clarke'schen Säulen), im peripherischen Nervensystem hochgradige parenchymatöse degenerative Neuritis mit interstitiellen Processen, und schliesslich bot die Muskulatur durch ihre sehr ausgedehnten und mannigfaltigen Veränderungen ein ganz besonderes interessantes Verhalten dar. Es wird jetzt unsere Aufgabe sein zu untersuchen, welche Bedeutung diesen einzelnen pathologischen Vorgängen zukommt, wie ihr Verhältniss zu einander aufzufassen ist, ob sich Etwas über den primären Sitz des Leidens eruiren lässt.

Dass wir Veränderungen in den peripherischen Nerven finden würden, war nach den klinischen Erscheinungen und bei Berücksichtigung des ätiologischen Momentes, des Alkohols zu erwarten. Das pathologisch-anatomische Bild der Neuritis ist seit der bekannten Arbeit Leyden's ${ }^{* * *}$ ) schon so oft Gegenstand ausführlicher Erörterungen gewesen, dass ich glaube, hier nur auf einen Punkt hinweisen zu dürfen, der von Interesse erscheint. Es ist dies das gleichzeitige Vorkommen von rein atrophischer parenchymatöser Degeneration mit

*) Dieses Archir Bd. XIX. S. 324.

**) Charité-Annalen XIV. Jahrgang.

**:*) Zeitschr. f. klin. Med. 1880. Bd. I. 
interstitiellen Processen am Bindegewebe und den Gefässen. Erb*) macht für die erstere, neurotische Atrophie, Veränderungen in den trophischen Centren des Rückenmarks verantwortlich, während er die interstitiellen Vorgänge, als wirklich periphere Neuritis auffasst. Diese Annahme führt uns sogleich zur Würdigung des Rückenmarkbefundes. Derselbe war unzweifelhaft positiv; wir fanden deutliche, wenn auch nicht sehr ausgedehute Veränderungen an den Ganglienzellen der Vorderhörner in quantitativer und qualitativer Hinsicht.

Es vermehrt also unsere Beobachtung die Zahl derjenigen Fälle, wo neben einer ausgedehnten Polyneuritis eine Affection des Rückenmarks anatomisch mit Sicherheit constatirt werden kann. In unserem Fall waren, wie in den Beobachtungen von Leyden**) und Oppenheim ${ }^{\text {* }}$ ), vornehmlich die Vorderhornganglienzellen betroffen. Es scheint nach neueren Beobachtungen, als ob auch Erkrankungen der weissen Substanz des Rückenmarks häufiger bei der multiplen Neuritis vorkommen, als man bisher anzunehmen geneigt war. Näher auf diesen interessanten Punkt hier einzngehen, würde mich zu weit führen; ich verweise anf die Zusammenstellung in der jüngst erschienenen Pal'schen $\dagger$ ) Arbeit, die auch ein ausführliches Verzeichniss der betreffenden Literatur giebt.

Die vorderen Wurzeln fanden wir in unserem Fall völlig intact, Diese Thatsache, dass eine anatomisch nachweisbarer Erkrankung der Ganglienzellen in den Vorderhörnern ohne degenerative Atrophie der vorderen Wurzeln, bei intensiver Degeaeration der peripherischen Nerven gefunden wurde, verdient wohl registrirt zu werden. Mein Fall scheint mir Aehnlichkeit mit dem von Eisenlohr $\dagger \dagger$ ) veröffentlichten zu baben. Hier ergab die anatomische Untersuchung ebenfalls starke degenerative Veränderung in den peripherischen Nerven und den Muskeln, bei normalem Verhalten der vorderen Wurzeln und in den Vorderhornzellen der Hals- und Lendenanschwellung leichte aber deutliche Veränderungen. Während nur für Eisenlohr dieser Fall eine positive Stütze für die Erb'sche Theorie von der degenerativen Atrophie ist, erblickt Strümpell dagegen in ihm eine weitere „erwünschte Bestätigung seiner Anschaunng, dass nämlich eine principielle Scheidung der Poliomyelitis und der multiplen Neuritis über-

*) Neurol. Centralb1. 1883. S. 481.

**) 1 . c.

***; Berliner klin. Woohensehr, 1890. No. 24.

†) Ueber multiple Neuritis. Wien 1891 .

t†) Neurol. Centralbl. 1884. No. 7 and 8. 
haupt gar nicht unter allen Umständen gerechtfertigt ist, dass vielmehr beide Affectionen unter einem einheitlichen ätiologischen Gesichtspunkte aufzufassen sind“.

Auf die Auffassung unseres, dem Eisenlohr'schen so ähnlichen Falles werden wir später zurückkommen.

Die Betheiligung der Muskulatur bei Alkoholneuritis ist durch die Arbeiten von Moeli*), Siemerling**), Oppenheim***), Eichhorst $\dagger$ ) u. A. gewürdigt und genauer beschrieben worden, so dass ich auf diese Untersuchungen verweisen darf. Die Veränderungen der Muskulatur boten in unserem Fall aber so viel Interessantes und Besonderes dar, dass wir auf diesen speciellen Befund näher eingehen müssen.

Das Bild, welches uns die Muskulatur zeigt, gleicht in ganz frappanter Weise der von Erb ${ }^{\dagger}$ ) in seiner grossen Arbeit gegebenen Schilderung der Veränderungen der Muskulatur bei Dystrophia muscularis progressiva. Wir fanden die von Erb so prägnant beschriebenen hypervoluminösen und atrophischen Fasern, „an Verbreitung, Zabl und Grösse ausserordentlich wecbselnd " mit der „charakteristischen Abrundung der Fasern"; ferner constatirten wir eine Vermehrung der Muskelkerne, eentral gelegene Kerne und Kernzeilen. Auch traten Spalt- und Vacuolenbildungen, sowie Fasertheilungen deutlich hervor. Gröbere Degenerationsvorgänge an den Muskelfasern selbst (fettige Degeneration, körnige Trübung, hyaline oder wachsartige Degeneration etc.) wurden nicht gefunden.

Die interstitiellen Veränderungen entsprechen ebenfalls durchaus der Erb'schen Schilderung, „Vermehrung und Wucherung des interstitiellen Bindegewebes mit reichlicher Kernanbäufung, Vermehrung und Verdickung der Gefässe, die ebenfalls zahlreiche Kerne enthalten und eine mehr oder weniger reichliche Ablagerung von Fettzellen in Reihen und Haufen."

Im Einzelnen bestehende kleine graduelle Unterschiede stimmen schliesslich auch durchaus mit der Beschreibung Erb's überein,

*) Charité-Annalen 1884. S. 541.

**) Dieses Archiv Bd. XVII. 1. - Charité-Annalen XIV. Jahrgang.

***) Berliner klin. Wochensohr. 1890. No. 24 (nach einem Vortrage gebalten in der Gesellschaft der (harité-Aerzte).

†) Virchow's Archiv Bd. CXII. S. 237.

(t) Deutsche Zeitschr. f. Nervenheilkunde. 1. Bd. 3. und 4. Heft. 
welcher die wechselvolle Mannigfaltigkeit des Gesammtbildes betont (vergl. Fig. 1, 2, 3 u. 4). Dass dieser Befund für mich höchst befremdend war, liegt auf der Hand, denn die klinische Beolachtung, wenn sie auch manches Unklare darbot, liess doch den Gedanken, dass es sich vielieicht um eine Dystrophie gehandelt hätte, nicht anfkommen. Gegen eine solche Annahme sprach das acute Entsteben des Leidens, die Lähmungserscheinungen und Sensibilitätsstörungen, die Entwickelung von Oedemen, das Entstehen einer hochgradigen allgemeinen Adipositas, schliesslich das Fehlen aller derjenigen Symptome, welche von Erb für die Dystrophie als charakteristisch hervorgeboben sind. Erb selbst giebt uns in seiner Arbeit (l. c.) einen Anhaltspunkt zur Erklärung dieses merkwürdigen Befundes, wenn er p. 244 sagt: „von ganz besonderem Interesse ist nun das Vorkommen ähnlicher Maskelveränderungen bei unzweifelhaft spinalen Erkranknngen." Er weist auf Fälle von $\mathrm{Pal}^{*}$ ) und Hitzig*; hin, in denen sich bei centralen Leiden Muskelhypertrophien fanden. Ferner hebt Erb Befunde von W. Müller*s; , Dejerine $\dagger$ ), Joffroy und Achard $\dagger$ ), Hitzigt十 ) (und Kawka), Oppenheim* ${ }^{*}$ ) und Fr. Schultze $\mathrm{e}^{* * *+}$ ) hervor, die bei verschiedenen spinalen Erkrankungen (spinalen Kinderlähmungen, Poliomyelitis ant. chron. etc.) in :den Muskeln Veränderungen fanden, die den bei Dystrophie beobachteten sehr ähnlich und zum Theil (Hitzig und $\mathrm{Kawka}$ ) ganz gleich waren. Erb und Hitzig erklären diese eigenthümlichen Muskelbefunde durch die Annahme, „dass einzelne restirende trophische Elemente (Ganglienzellen? Fasernetze?) unter den gegebenen pathologischen Bedingungen eine Ernährungsstörung in den Muskeln hervorrufen, die mit derjenigen bei Dystrophie eine grosse Aehnlichkeit hat, dass also eine gestörte und nicht vollkommen aufgehobene Function dieser Elemente zu einer greifbaren trophischen Störung in den Muskeln führt“.

*) Wiener kin. Wochensehr. 1889. No. 10.

*s) Berliner klin. Wochensohr. 1888.

***) Beitrag zur patholog. Anatomio und Physiologie des Rüokenmarks. Leipzig 1871.

b) Compt. rend. de Soc. de Biol. 1887. 19. Mars.

th) Archives de Physiol. norm. et path. 1888. I. p. 375.

H†) Hitzig, Berliner klin. Wochonsohrift 1889. No. 28. - Kawka, Bẹträge zur patbologischen Anatomie der spinalen Kinderlähmung. Dissert. Halle 1889.

*) Dieses Archiv Bd. XIX. S. 381.

†) Zeitschr. für kllin. Medicin Bd. XIII. 
Jch wollte hier nur meinen Befund den von Erb als „höchst bemerkenswerth, wenn auch selten und ausnahmsweise c bezeichneten Beobachtungen anreihen.

Fragen wir nun nach dem primären Sitz des Leidens, ob das Rückenmark, die peripherischen Nerven oder die Muskeln zuerst erkrankt sind, so treten uns bei der Beantwortung dieser Frage die grössten Schwierigkeiten entgegen. Wohl unzweifelhaft haben wir es in unserem Fall mit der Einwirkung einer bestimmten toxischen Sub$\operatorname{stan} z$ - des Alkohols - auf den Organismus zu thun und wir werden von vornherein die Möglichkeit zugeben müssen, dass diese Noxe die Punkte des neuromusculären Tractus einzeln oder mehrere zu gleicher Zeit direct angreifen kann. Zur Entscheidung der Frage, welche Stelle die zuerst erkrankte ist, könnte vielleicht der anatomische Befund in ganz frischen, nach kurzem Krankheitsverlauf letal endenden Fällen herangezogen werden. Aber auch in diesen seltenen $\mathrm{Be}-$ obachtungen werden uns gewichtige $Z$ weifel an der Zuverlässigkeit der anatomischen Methode entgegen treten, denn es können ja nach E rb in der Peripherie anatomisch nachweisbare atrophische Zustände eintreten, unter dem Einfluss in ihrer Function gestörter, anatomisch noch nicht nachweisbar erkrankter Ganglienzellen. Wie viel schwieriger ist die Entscheidung der Frage aber erst in Fällen, die Jahre lang bestimmten toxischen Einflüssen ausgesetzt sind, in denen schwere Krankheitserscheiuungen lange Zeit bestanden haben, wie bei unserer Beobachtung. Lässt uns ja die Schwere und Ausdehnung der anatomischen Veränderung an einer bestimmten Stelle nicht auf das Alter der Störung schliessen, denn hier spielen sicherlich individuelle Verhältnisse, wie verschiedene Resistenzfähigkeit der einzelnen Gewebe gegen toxische Einwirkungen eine nicht zu unterschätzende Rolle. Das häufige Intactbleiben der vorderen Rückenmarkswurzeln bei sicheren Veränderungen in den Vorderhörnern und an der Peripherie, könnte möglicherweise auch in einer solchen grösseren Widerstandskraft gegen Noxen eine Erklärung finden. Selbstverständlich sind das nur Hypothesen, die wir aber zunächst bei der Erklärung so complicirter Processe kaum entbehren können. Wenden wir diese Ueberlegungen auf unseren Fall an, so werden wir, da uns die klinische Beobachtung keinen sicheren Anhaltspunkt giebt, die Frage, wo das Leiden begonnen hat und in welcher Weise es fortgeschritten ist, nicht entscheiden können. Zwei Möglichkeiten scheinen mir vornehmlich ins Auge gefasst werden zu müssen.

1. Der Alkohol hat zunächst seine deletäre Wirkung auf die 
trophische Centren in den Vorderhörnern des Rückenmarks ausgeübt, hier Veränderungen an den Ganglienzellen bervorgerufen. Secundär sind dann zuerst die peripherischsten Stellen, die Muskeln und die feinsten peripherischen Nerven erkrankt, die in der That hochgradigste Degeneration erkennen liessen. Dann ist der Process centralwärts fortgeschritten, hat zur Degeneration der stärkeren Nervenäste geführt, die vorderen Wurzeln hingegen noch verschont.

2. Die Noxe hat die Ganglienzellen, Nerven und Muskeln direct nacheinander oder gleichzeitig angegriffen und an den locis minoris resistentiae die ausgedehntesten, an resistenzfähigeren Stellen schwăchere Veränderungen hervorgerufen.

Anmerkung. Die erste Aunahme findet eine Stütze in der schönen experimentellen Arbeit von Stieglitz (Archiv f. Psych. u. Nervenkrankh., Bd. XXIV., Heft 1), welche mir erst nach Vollendung des Manuscripts zur Kenntniss gekommen ist. In dieser Arbeit wurden die Voraussetzungen, auf welchen die Theorie Erb's von der functionellen Beeinträchtigung der spinalen Centren bei der Bleilähmung beruhen, durch pathologisch-anatomische Thatsachen bewiesen. Fs ist jedenfalls nicht von der Hand zu, weisen, dass die Thatsachen, welche Stieglitz für die secundäre Degeneration bei Bleiintoxication festgestellt hat, auch bei anderen Intoxicationen, z. B. denen mit Alcohol, Geltung behalten könnten.

Schliesslich möchte ich noch erwähnen, dass die Eigenthümlichkeiten in dem klinischen Verlauf (rasche Entwickelung der enormen Obesitas, die Deformitäten der Hände etc.) durch den anatomischen Befund nicht erklärt werden. Die psychischen Veränderungen sind wohl durch den chronischen Alcoholismus bedingt worden.

Herrn Geh.-Rath Jolly bin ich für die freundliche Ueberlassung des Materials zu aufrichtigem Dank verpflichtet.

Herrn Prof. Dr. Siemerling spreche ich für die liebenswürdige Hülfe, welche er mir bei dieser Untersuchung hat zu Theil werden lassen, hier meinen besten Dank aus. 


\section{Erklärung der Abbildungen (Tafel XIII. und XIV.).}

\section{Tafel XIII.}

Fig. 1, 2, 3. M. vastus fem. Querschnitt.

Färbung: Hämatoxylin (Z eiss B. Oc]. 3. [1:115]).

Fig. 4. M. interosseus (Manas). Quersehnitt.

Färbung: Carmin, Hämatoxylin (Z eiss B. Ocl. 3. [1:115]).

Fig. 5. N. tibialis post. Querschnitt.

Färbung in Weigert (Zeiss B. Ocl. 3. [3:115]).

Fig. 6. Ganglienzellen aus einem Vorderhorn der Halsanschwellung.

Färbung: Carmin (Zeiss D. Ocl. $1[1: 175]$ ).

\section{Tafel XIV.}

Fig. 7. Schematische Uebersicht der Zahl der Ganglienzellen in einem Vorderhorn (Halstheil) bei Frombolz.

Fig. 8 in einem normalen Vorderborn.

Zeiss a. Ocl. (1:15). 
$\therefore \quad \therefore$ $\therefore \div \quad 3$

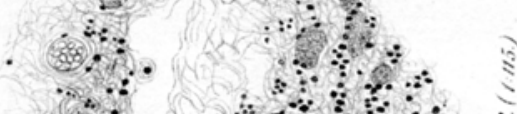

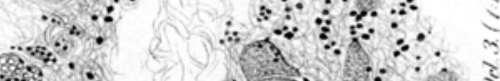

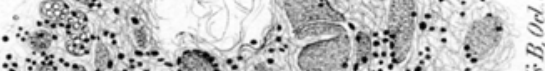

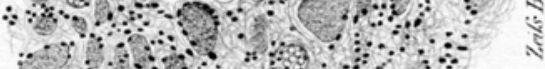

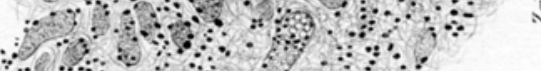
$\begin{array}{lll}3 & 40 \% \\ 3 & 9\end{array}$
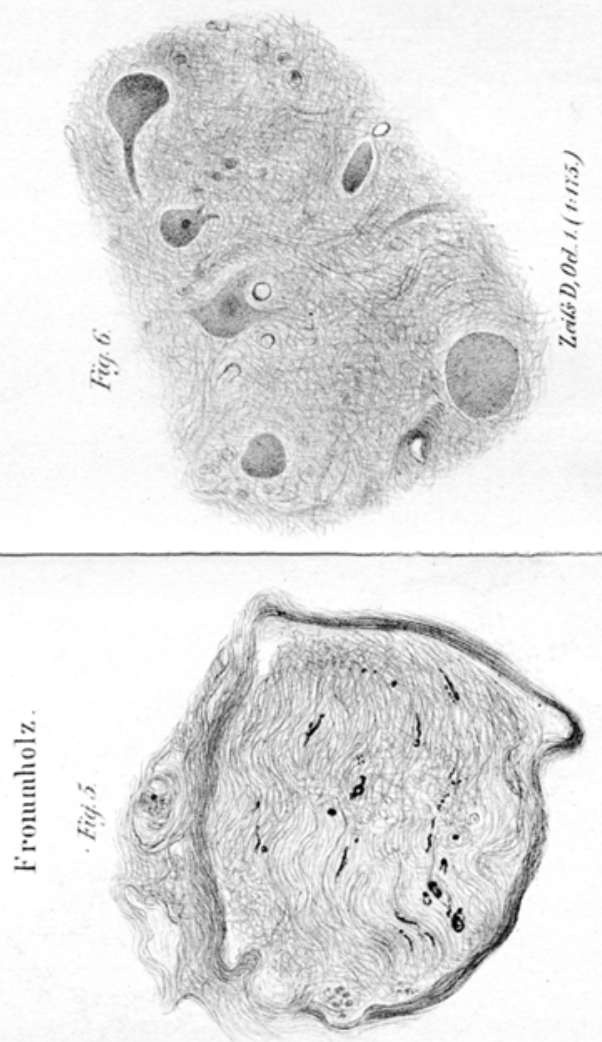

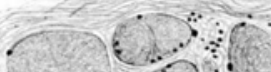

(a) $\therefore$ :
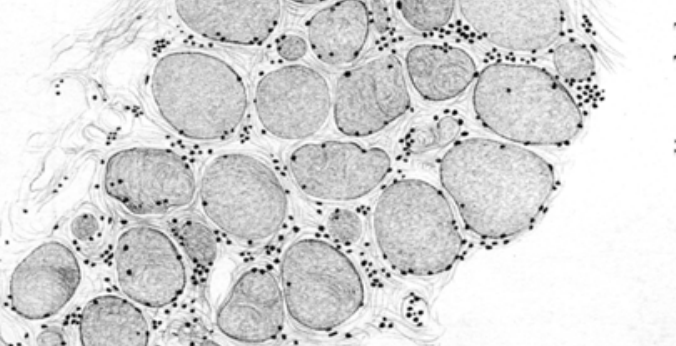

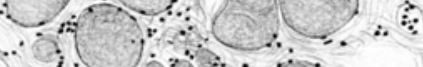

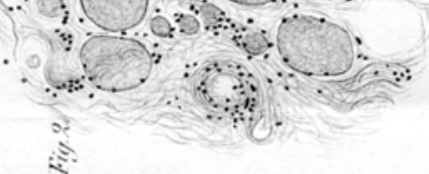

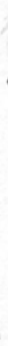

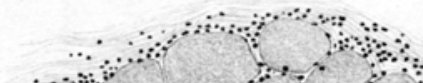

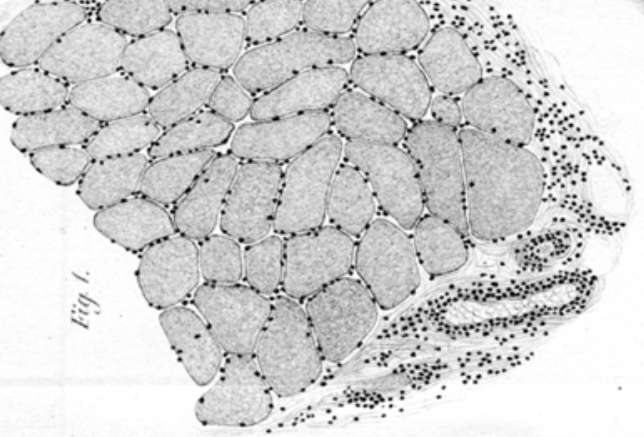
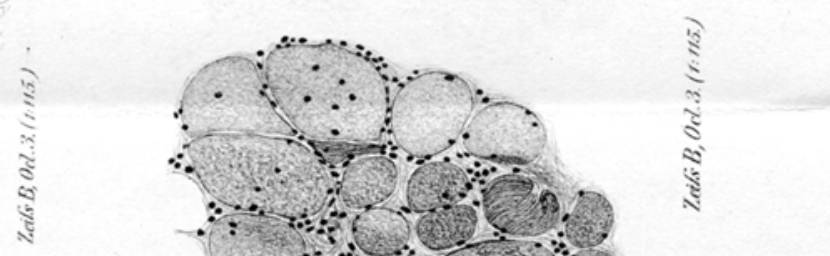
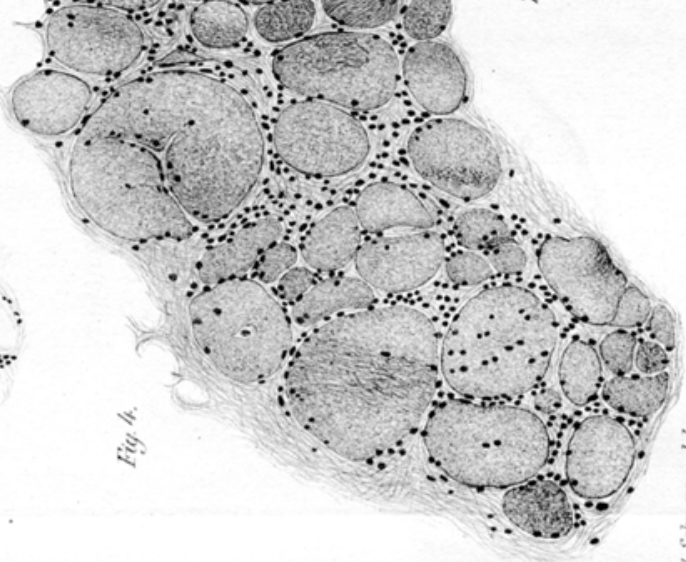
Frommholz.

Fig. $\%$

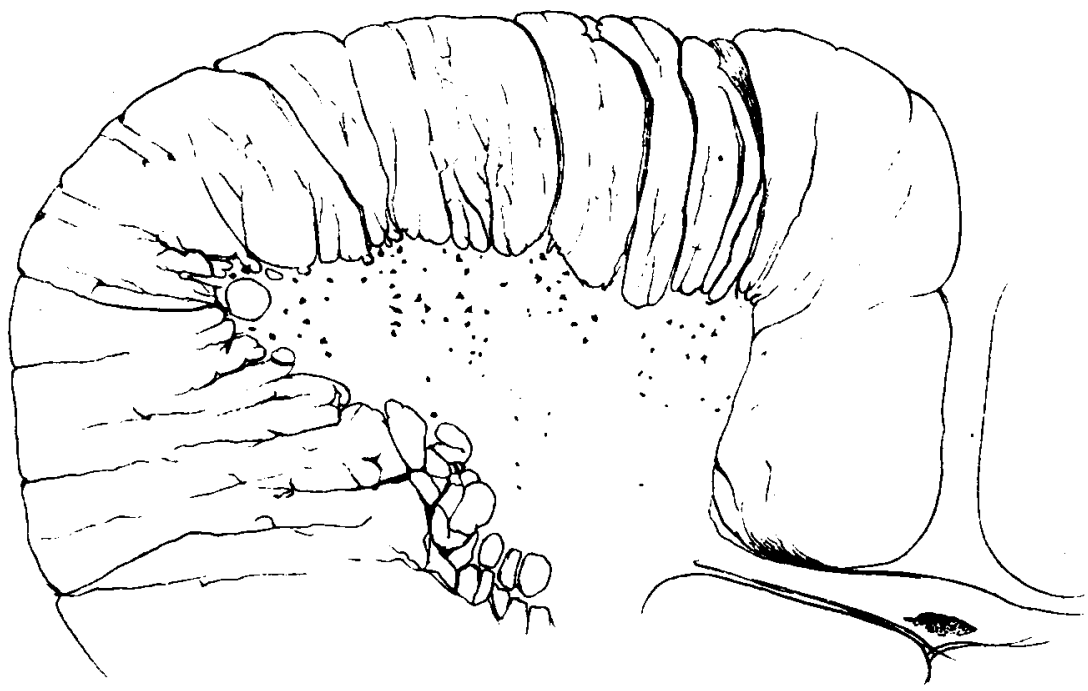

Fig.8.

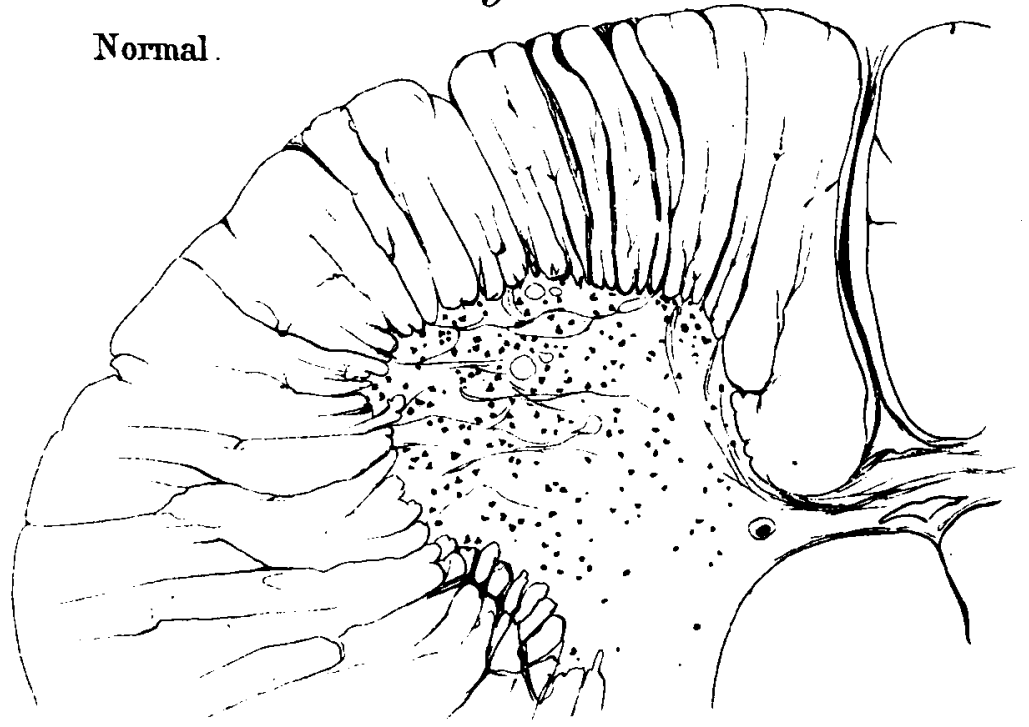

Zaifa, Od.3. (1::5.) 